Congress in November 2000 mandating OSHA to enforce the use of sharps safety devices. The effective date of the revised regulation is April 18, 2001. States with their own occupational safety plans have 6 months to adopt a comparable standard. If a state has a stricter needle safety law already in place, the state law will still apply. The only hospitals not covered under the rule are public hospitals in states without their own occupational health and safety agency.

The revisions include a new definition of an engineering control to include as examples, a device with engineering sharps-injury protection, and a needleless IV device. In addition, there must be an annual review and revision of the exposure control plan to include consideration appropriate, commercially available, and effective safer medical devices to reduce risk of exposures. An appropriate safer medical device is one that would not jeopardize patient or employee safety or be medically contraindicated, the rule states. The plan must include a description of the devices, methods used to evaluate them, and reasons for adoption. Employers are also required to include frontline workers in the identification, selection, and evaluation of these devices, and to document this input in the plan. Also, the revised rule requires a sharps-injury log that collects information on the type and brand of device involved in the incident, where the incident occurred, and an explanation of the incident with adequate privacy protection.

OSHA also referred to the publication of OSHA's final recordkeeping rule, which was published separately. This revised recordkeeping rule introduced new forms for recording occupational injuries and illnesses, but noted that the detailed sharps-injury log must be kept in accordance with the revised Bloodborne Pathogen Standard.

OSHA has commented that this revised rule does not change the enforcement of the use of sharps safety devices, which were already clarified in the revised November 1999 Enforcement Procedures for the Bloodborne Pathogen Standard. The January 18, 2001, revision only formalizes this. OSHA has been citing hospitals for lack of use of sharps safety devices since November 1999 . There has been some flexibility in the citations issued for employers that have evidence of adoption of some devices and a detailed plan outlining the plan for completion with timelines.

FROM: Department of Labor. OSHA. Occupational exposure to bloodborne pathogens: needlestick and other sharps injuries. Final rule. Federal Register 2001;66:53175325.

\section{JCAHO Approves New Patient Safety Standards}

The Joint Commission on Accreditation of Healthcare Organizations (JCAHO) has approved standards directly focused on patient safety and medical error reduction in hospitals. The implementation date for the standards is expected to be July 2001. The new standards expand on current JCAHO standards, which require healthcare organizations to identify, report internally, and analyze sentinel events and to take action to prevent their recurrence. Requirements for patient-safety programs will be added in the following areas:

Leadership: Hospital leaders are to encourage error identification and remedial steps to prevent future errors. Individual blame or retribution should be minimized for those involved in an error or in reporting an error.

Improving organization performance: Hospitals are to implement a program for proactive assessment of high-risk activities related to patient safety and to undertake appropriate improvements. The hospital will select which activities to assess based on available knowledge, including information that is provided by JCAHO through its study of adverse events that seriously harm patients (sentinel events).

Information management: Patient-safety-related data should be aggregated by the hospital to identify risk to patients, and results should be communicated effectively to caregivers and others involved in patient safety to reduce these risks.

Other functions: Patient safety should be emphasized in areas such as patient rights, education of patients and their families, continuity of care, and human resources management. The patient or the patient's family should be informed about the results of care, including unanticipated outcomes.

FROM: icanNEWS. January 2, 2001. http://www. icanprevent.com.

\section{US Action Plan to Combat Antimicrobial Resistance}

An action plan, developed by an interdepartmental task force, was unveiled recently that provides the United States with a comprehensive approach to combat antimicrobial resistance. The plan designates priorities and identifies responsible agencies and timelines.

The CDC, the NIH, and the FDA led a task force of 10 agencies and departments that included the Agency for Healthcare Research and Quality, the Health Care Financing Administration, and the Health Resources and Services Administration at HHS, as well as representatives from the Department of Agriculture, the Department of Defense, the Department of Veterans Affairs, and the EPA.

The plan has four major components: surveillance, prevention and control, research, and product development. Top priorities of the four major sections include:

Surveillance. The CDC will work with state health departments and other task force members to design and implement a plan that will define national, regional, state, and local antimicrobial-resistance surveillance responsibilities, so that these entities are coordinated and use similar methodology. Additionally, systems will be developed that can monitor patterns of antimicrobial-drug use in human medicine, in agriculture, and in consumer products.

Prevention and control. A national public education campaign will be launched to reduce the overuse and 
misuse of antimicrobial drugs and to improve antibiotic use in healthcare systems. Along with professional societies and other stakeholders, the CDC already has started to prepare clinical guidelines for health professionals on how best to use antimicrobials. Additionally, the FDA has initiated consultations with stakeholders to refine its proposed framework for assessing the human health impact of antimicrobials that may be used in foodproducing animals.

Research. Research will be provided to the community with new information and technologies, including genetic blueprints for various microbes, to identify targets for desperately needed new diagnostics, treatments, and vaccines that could assist in preventing the emergence and spread of resistant pathogens. The NIH plans to develop clinical studies to test new antimicrobials and novel approaches to treating and preventing infections caused by resistant pathogens. The NIH continues to encourage and facilitate new rapid diagnostic methods and will pursue their development and evaluate their ultimate impact in the context of antimicrobial resistance.

Product development. To identify and publicize priority health needs for new products that prevent resistance or treat resistant infections, HHS plans to create an Interagency Antimicrobial Product Development Working Group. Once formed, this group also will consult with stakeholders and economic consultants to identify incentives that encourage this kind of product development.

FROM: Centers for Disease Control and Prevention. A Public Health Action Plan to Combat Antimicrobial Resistance. CDC's antimicrobial resistance web site, http://www.cdc.gov/drugresistance.

\section{VRE Colonization in Liver and Kidney Transplant Recipients}

At Mayo Medical Center (Rochester, MN), surveillance rectal (and other-site) cultures have been collected routinely from liver transplant recipients as part of a selective bowel decontamination program. Beginning in 1995, vancomycin-resistant Enterococcus (VRE) colonization and infection were identified in Mayo Clinic liver and kidney transplant patients through our surveillance cultures. Patel and colleagues conducted a study to describe the natural history of VRE colonization in this patient population. Fifty-two patients with VRE colonization (predominantly with a single $\operatorname{van} \mathrm{B}$ clone) were identified from September 1995 through December 1997. Five hundred ninety cultures were reviewed for this study (mean, 11.3 cultures/patient). The median time from initial VRE colonization to the last surveillance culture obtained was 306 (range, 1-1,393) days.

VRE infection was documented in 6 patients (11.3\%). Eighteen patients (35\%) met the criteria for clearance of VRE colonization, defined as VRE-negative rectal-culture results on at least three consecutive occasions greater than 1 week apart. However, VRE was detected on sub- sequent surveillance cultures from 2 of these patients (11\% relapse rate). Of the remaining 34 patients, 16 remained colonized with VRE, and 18 did not meet the definition for clearance of VRE colonization because of incomplete follow-up.

This study documents that VRE colonization usually persists for months to years in liver and kidney transplant patients.

FROM: Patel R, Allen SL, Manahan JM, Wright AJ, Krom RA, Wiesner RH, et al. Natural history of vancomycin-resistant enterococcal colonization in liver and kidney transplant recipients. Liver Transpl 2001;7:27-31.

\section{VRE Among Chronic Hemodialysis Patients}

D'Agata and coinvestigators from Vanderbilt University School of Medicine, Nashville, Tennessee, conducted a study to determine the prevalence and rate of acquisition of vancomycin-resistant enterococci (VRE) among patients undergoing chronic (ie, long-term) hemodialysis who were admitted to a tertiary-care center. Serial rectal cultures for VRE were performed at hospital admission and every 5 days until hospital discharge. A total of $7(6 \%)$ of the 119 patients were colonized with VRE at admission. Six (19\%) of the 32 patients who remained in the hospital $\geqslant 4$ days acquired VRE.

A nonambulatory status was significantly associated with colonization at admission (odds ratio, 9.7; 95\% confidence interval $\left.\left[\mathrm{CI}_{95}\right], 1.8-53 ; P=.01\right)$, and vancomycin exposure was significantly associated with VRE acquisition (relative risk, $1.8 ; \mathrm{CI}_{05}, 1.1-2.9 ; P=.02$ ). All patients acquired VRE from epidemiologically linked dialysis patients colonized with similar VRE genotypes. Hospital acquisition of VRE contributes substantially to the increasing prevalence of VRE in the chronic hemodialysis patient population.

FROM: D'Agata EM, Green WK, Schulman G, Li H, Tang YW, Schaffner W. Vancomycin-resistant enterococci among chronic hemodialysis patients: a prospective study of acquisition. Clin Infect Dis 2001;32:23-29.

\section{Typing of Coagulase-Negative Staphylococci From Blood Cultures}

Seo and coinvestigators from Beth Israel Deaconess Medical Center and Harvard Medical School, Boston, conducted a study to determine whether a blood culture that contains coagulase-negative staphylococci represents bacteremia or contamination. They compared moleculartyping results of coagulase-negative staphylococcal blood culture isolates with clinical criteria for true bacteremia. Pulsed-field gel electrophoresis and arbitrarily primed (AP) polymerase chain reaction (PCR) were used to determine whether patients with two or more blood cultures with coagulase-negative staphylococcal isolates had the same strain of organism in each culture (same strain bacteremia). They evaluated three different clinical criteria for bacteremia: whether the patient received more than 4 days 